\title{
Pseudomonas aeruginosa PAO-1 Lipopolysaccharide-Diphtheria Toxoid Conjugate Vaccine: Preparation, Characterization and Immunogenicity
}

\author{
Faezeh Najafzadeh ${ }^{1}$; Reza Shapouri ${ }^{2}$; Mehdi Rahnema ${ }^{3}$; Shadi Rokhsartalab Azar ${ }^{4}$; \\ Anvarsadat Kianmehr ${ }^{5,}$ \\ ${ }_{1}^{1}$ Young Researchers Club, Bonab Branch, Islamic Azad University, Bonab, IR Iran \\ ${ }^{2}$ Department of Microbiology, Zanjan Branch, Islamic Azad University, Zanjan, IR Iran \\ 3 Biologic Research Center, Zanjan Branch, Islamic Azad University, Zanjan, IR Iran \\ ${ }_{5}^{4}$ Young Researchers and Elite Club, Urmia Branch, Islamic Azad University, Urmia, IR Iran \\ ${ }_{5}$ Young Researchers and Elite Club, Urmia Branch, Islamic Azad University, Urmia, IR Iran \\ ${ }^{*}$ Corresponding author: Anvarsadat Kianmehr, Department of Medical Biotechnology, School of Advanced Medical Sciences, Tabriz University of Medical Sciences, Tabriz, IR Iran.
} Tel:+98-2166402770, Fax:+98-2166465132, E-mail: kiabiotpro@yahoo.com

Received: January 18, 2014; Revised: May 26, 2014; Accepted: June 7, 2014

Background: Treatment of Pseudomonas aeruginosa PAO-1 infections through immunological means has been proved to be efficient and protective.

Objectives: The purpose of this study was to produce a conjugate vaccine composed of detoxified lipopolysaccharide(D-LPS) P. aeruginosa and diphtheria toxoid (DT).

Materials and Methods: Firstly, LPS was purified and characterized from P. aeruginosa PAO1 and then detoxified. D-LPS was covalently coupled to DT as a carrier protein via amidation method with adipic acid dihydrazide (ADH) as a spacer molecule and 1-ethyl-3(3-dimethylaminopropyl) carbodiimide (EDAC) as a linker. The molar ratio of LPS to DT in the prepared conjugate was 3:1. The immunogenicity of D-LPS-DT conjugate vaccine in mice model was evaluated as well.

Results: The conjugate was devoid of endotoxin activity and $0.125 \mathrm{U} / \mathrm{mL}$ of D-LPS was acceptable for immunization. D-LPS-DT conjugate was nonpyrogenic for rabbits and nontoxic for mice. Mice immunization with D-LPS-DT conjugate vaccine elicited the fourfold higher IgG antibody compared to D-LPS. Anti-LPS IgG antibody was predominantly IgG1 subclass and then IgG3, IgG2a and IgG2b, respectively. Conclusions: Vaccine based on the conjugation of P. aeruginosa PAO-1 LPS with DT increased anti-LPS antibodies and had a significant potential to protect against Pseudomonas infections.

Keywords: Pseudomonas aeruginosa PAO-1; Vaccines, Conjugate; Lipopolysaccharides; Diphtheria Toxoid

\section{Background}

Pseudomonas aeruginosa is one of the most common nosocomial pathogens due to many factors including emergence of antibiotic resistant strains and production of several virulence factors $(1,2)$. This bacterium causes life-threatening infections, especially in individuals with cystic fibrosis, severe burns or immunocompromised patients. The Infections caused by this pathogen are associated with highest mortality rate in hospitalized patients and difficult to treat (3). Treatment of $P$. aeruginosa infections is greatly hampered by innate and acquired antibiotic resistance (4). In fact, this pathogen has more capability for circumventing the activities of antimicrobials than any other microorganism. These findings have served as an impetus to investigate the feasibility to control $P$. aeruginosa infections through immunological means (5). Lipopolysaccharide (LPS) is the major surface antigen of this bacterium playing an important role in the interaction with the host immune system.
Human immunity to $P$. aeruginosa has been correlated with humoral antibody to LPS. Anti-LPS antibody has been shown to be highly protective against $P$. aeruginosa infections in many animal models (6). LPS is a thymusindependent antigen, which induces an immune response without involvement of T-helper lymphocytes. Polysaccharides induce a poor response in infants, and at later ages of life, the responses are of short duration and cannot be boosted. To overcome these problems, polysaccharides must be converted to thymus-dependent antigen by conjugation to a carrier protein to create effective vaccines (7). Diphtheria toxoid (DT) has been used as protein carrier to enhance the immunogenicity of LPS (8).

\section{Objectives}

The purpose of this study was to produce a conjugate vaccine by coupling detoxified P. aeruginosa PAO-1 LPS to DT. 


\section{Materials and Methods}

\subsection{Bacterial Strain and LPS Preparation}

Pseudomonas aeruginosa PAO1 was obtained from the Biologic Research Center, Islamic Azad University, Zanjan Branch, Iran. It was grown in nutrient broth medium in shaker incubator at $37^{\circ} \mathrm{C}$ for 72 hours (9). LPS was extracted by the hot phenol procedure with some modifications. Cells were suspended in water and heated at $66^{\circ} \mathrm{C}$ for 20 minutes, then $90 \%$ phenol was added and the mixture was stirred at $66^{\circ} \mathrm{C}$ for 30 minutes. The resulting mixture was rapidly cooled by stirring on ice. The suspension was centrifuged at $4000 \mathrm{rpm}$ at $4{ }^{\circ} \mathrm{C}$ for 45 minutes. The aqueous phase was removed and 95\% cold ethanol with $50 \%$ final concentration was added to precipitate the proteins and nucleic acids. After centrifugation, trichloroacetic acid (TCA) with 10\% final concentration was added and stirred at $4^{\circ} \mathrm{C}$ for 30 minutes. Following centrifugation, the supernatant was dialyzed against distilled water at $4{ }^{\circ} \mathrm{C}$ for 24 hours and LPS was precipitated with three volumes of cold $100 \%$ ethanol and placed at $4^{\circ} \mathrm{C}$ overnight. The suspension was centrifuged and the pellet was collected. Purified LPS was lyophilized and stored at $4^{\circ} \mathrm{C}$. Sodium dodecyl sulfatepolyacrylamide gel electrophoresis (SDS-PAGE) was used for LPS characterization. LPS bands were stained and visualized by silver staining method. The protein content of LPS was determined by the Bradford method with bovine serum albumin (BSA) as a standard. Nucleic acid was also estimated by measuring the absorbance at $260 \mathrm{~nm}$.

\subsection{Detoxification of LPS}

LPS pellet was dissolved in $0.2 \mathrm{~N} \mathrm{NaOH}$ and heated at $100^{\circ} \mathrm{C}$ for two hours. Free fatty acids were removed by dialyzing extensively against distilled water at $4^{\circ} \mathrm{C}$ for two days with six changes of water. Three volumes of cold ethanol were added to the mixture and then placed at $4^{\circ} \mathrm{C}$ overnight. The suspension was centrifuged at 4000 grams at $4^{\circ} \mathrm{C}$ for 45 minutes (10). The level of endotoxin in D-LPS was assayed by the Limulus amoebocyte lysate (LAL) test and expressed in endotoxin units related to the U.S. standard (11).

\subsection{Rabbit Pyrogen Test for D-LPS}

The rabbit pyrogen test was performed for D-LPS. Briefly, $10 \mu \mathrm{g}$ D-LPS was injected to rabbits weighing 2.5 to 3 kilograms. Each group consisted of three rabbits. The baseline rectal temperature was recorded every 15 minutes for one hour before the injection. After one hour, rabbits were inoculated in the ear marginal vein. Temperatures were recorded every 15 minutes for three hours after the injection. The test indicates the presence of a pyrogen when one of the three rabbits shows an increase in body temperature higher than $0.5^{\circ} \mathrm{C}$ or when the sum of the maximum temperature rises in the three individual rabbits exceeds $1.2^{\circ} \mathrm{C}(10)$.

\subsection{Synthesis and Characterization of D-LPS-DT Conjugate}

D-LPS was covalently coupled to DT as a carrier protein via amidation method with adipic acid dihydrazide $(\mathrm{ADH})$ as a spacer molecule and 1-ethyl-3-(3-dimethylaminopropyl) carbodiimide (EDAC) as a linker. Briefly, $\mathrm{NaCNBH} 4(0.1 \mathrm{~g})$ and acetonitrile $(0.5 \mathrm{~mL})$ were added to D-LPS and the reaction mixture was stirred at $4^{\circ} \mathrm{C}$. After 10 minutes of incubation, an equal volume of $0.5 \mathrm{~N}$ $\mathrm{ADH}$ in $0.5 \mathrm{~N} \mathrm{NaHCO} 3$ was added and $\mathrm{pH}$ was adjusted to 8.5 with $0.1 \mathrm{~N} \mathrm{HCl}$. The reaction was performed at $4^{\circ} \mathrm{C}$ for 18 hours and then dialyzed against water at $4^{\circ} \mathrm{C}$ for 24 hours with three changes of water. After that, $4 \mathrm{~mL}$ DT containing $10 \mathrm{mg} / \mathrm{mL}$ protein and $0.1 \mathrm{~N}$ EDAC was added to the conjugation reaction and then stirred on ice for four hours. The reaction mixture was dialyzed against distilled water at $4^{\circ} \mathrm{C}$ for two days with six changes of the outer fluid. The D-LPS-DT conjugate product was purified by gel filtration method using CL-2B Sepharose column $(1.5 \times 90 \mathrm{~cm})$. Fractions with the maximum absorbance for both protein and polysaccharide were pooled, dialyzed and then lyophilized under aseptic conditions (12). The protein content of D-LPS-DT conjugate was estimated by the method of Bradford, with BSA as a standard. Carbohydrate content of conjugate was also measured by the phenol-sulfuric acid method, with glucose as a standard (13).

\subsection{Rabbit Pyrogen Test}

Rabbit pyrogen test for D-LPS-DT conjugate was performed as previously described for D-LPS in Section 3.3.

\subsection{Toxicity Test}

The lethal effect of D-LPS-DT conjugate vaccine was evaluated in five mice. One human dose of conjugate (10 $\mu \mathrm{g} / \mathrm{mL}$ ) was administered intraperitoneally to each mice. Mice were monitored for weight change and mortality for seven days post-challenge (12).

\subsection{Sterility Test}

Conjugate was cultured in Thioglycollate, Nutrient agar, Blood agar, Macconkey agar and sabouraud dextrose agar mediums. Media were incubated in aerobic and anaerobic conditions and observed after incubation at $37^{\circ} \mathrm{C}$ for 24 to 48 hours (3).

\subsection{Immunogenicity Studies}

Sixty female BALB/c mice ( 6 - 8 weeks old) were immunized intraperitoneally in four groups of fifteen on days 
0,14 and 28 with either $10 \mu \mathrm{g}$ of D-LPS, DT and D-LPS-DT conjugate. Control treatment was also prepared by mixing conjugate solutions with the same volume of normal saline. Venous blood samples were obtained on days 14 , 28 and 42 . Sera were collected by centrifugation at 2500 rpm for 15 minutes (11).

\subsection{Enzyme Linked Immunosorbent Assay (ELISA)}

Antibody response levels against D-LPS, D-LPS-DT and DT were determined by ELISA method for total IgG, IgA, IgG1, IgG2a, IgG2b and IgG3. DT sample solution $(2 \mu \mathrm{g} / \mathrm{mL}$ in $0.05 \mathrm{M}$ sodium carbonate buffer, $\mathrm{pH}$ 9.6) was coated into ELISA plate and incubated at $4^{\circ} \mathrm{C}$ for overnight. Blocking buffer was added and incubated for one hour at room temperature. Mouse sera were diluted in PBS (1:10) and assayed in triplicate. Incubation was performed for two hours at room temperature. Horseradish peroxidase-conjugated goat anti-mouse IgG (diluted 1: 3000 in 1\% BSAPBS) was added and incubated at room temperature for one hour. PBS containing 0.05\% Tween 20 (PBST) was used for washing (3X) between steps. O-Phenylenediamine dihydrochloride and $\mathrm{H}_{2} \mathrm{O}_{2}$ were used as substrate. After 15 minutes incubation in dark, the reaction was stopped by addition of $50 \mu \mathrm{L} 2 \mathrm{~N} \mathrm{H}_{2} \mathrm{SO} 4$ and absorbance at 450 nm was measured (11). Antibody response levels against D-LPS, D-LPS-DT and DT for IgM were determined as described above with one modification. For IgM, Goat antimouse antibody (diluted 1:1000 in 1\% BSA-PBS) was added as primary antibody and incubated for one hour at room temperature.

\subsection{Statistical Analysis}

Antibodies sera titers are expressed as a geometric mean. Comparison of geometric means was performed by SPSS 16.0 software using One-Way ANOVA test (Tukey) by $\mathrm{P}<0.01(12)$.

\section{Results}

\subsection{Characterization of Pseudomonas aeruginosa LPS}

SDS-PAGE gel followed by silver staining was applied to detect and visually characterize the purified LPS. Sliver staining is a highly sensitive technique capable of detecting as low as $1 \mathrm{ng}$ LPS. The results of SDS-PAGE electrophoresis showed purified $P$. aeruginosa LPS as a single dense band at the bottom of gel (Figure 1). As depicted in Figure 1, lanes 1 and 2 show 10 and $5 \mu \mathrm{g}$ of extracted LPS, respectively, and also no stained material was seen on the gel. The obtained LPS contained $1 \mathrm{mg} / \mathrm{mL}$ protein and $1 \mu \mathrm{g} / \mathrm{mL}$ nucleic acid. In the LAL test, D-LPS contained $0.125 \mathrm{EU} / \mathrm{mL}$, which was acceptable for immunization. The rabbit thermal induction test with $10 \mu \mathrm{g}$ of D-LPS showed no increase in body temperature. Data showed that the purified LPS was active and remained in its correct form with all epit- opes, so it could be used to inject into animals and induction of antibodies.

Figure 1. Silver Nitrate-Stained SDS-PAGE of Extracted Pseudomonas aeruginosa LPS

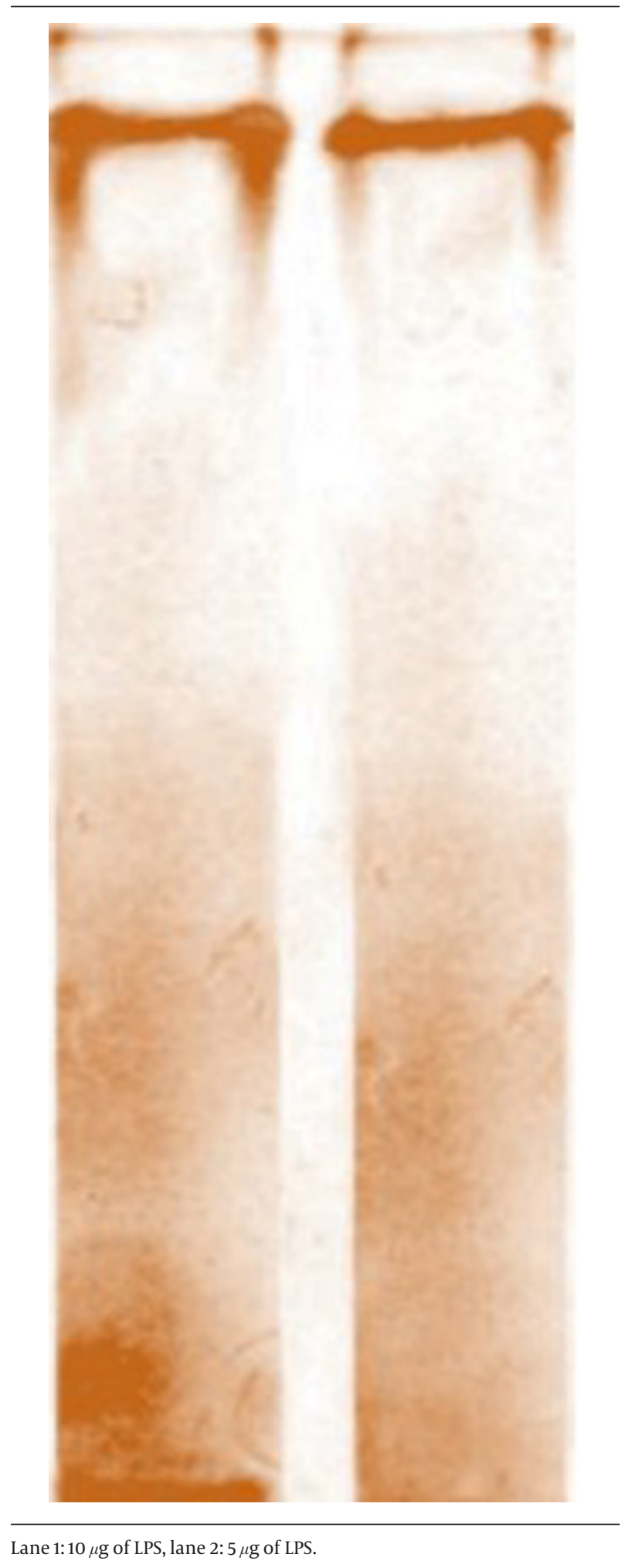




\subsection{Characterization of the Conjugate Vaccine}

The gel filtration profile of the D-LPS-DT conjugate through a CL-2B Sepharose column is shown in Figure 2. As seen, positive fractions (fractions 72 to 74 ) for polysaccharide and protein appeared as a single peak, indicating the D-LPS conjugation to DT. First minor peaks at both 210 and $280 \mathrm{~nm}$ showed D-LPS-D-LPS and DT-DT conjugate molecules, respectively, and second minor peaks at both 210 and $280 \mathrm{~nm}$ indicated D-LPS and DT non-conjugate molecules, respectively (Figure 2). The molar ratio of LPS to protein for D-LPS-DT conjugate was 3:1 and the labeling efficiency was estimated to be $43 \%$. The protein content of purified D-LPS-DT was $1 \mathrm{mg} / \mathrm{mL}$ protein. The carbohydrate assayed by the phenol-sulfuric acid method determined to be $0.4 \mathrm{mg} / \mathrm{mL}$.

\subsection{Evaluating Pyrogenicity, Toxicity and Sterility of D-LPS-DT Conjugate}

The D-LPS-DT conjugate was non-pyrogenic when tested at a dose of $10 \mu \mathrm{g}$ and evoked below $0.5^{\circ} \mathrm{C}$ increase in body temperature of each rabbit after 24 hours. There were no overt signs of toxicity after intraperitoneal administration of conjugate vaccine to mice. Mediums were observed after incubation at $37^{\circ} \mathrm{C}$ for 24 to 48 hours. No evidence of microbial growth was found. Sterility test showed that the resulting conjugate was sterile. P Values (probability of error) for all tests were less than 0.05 , which were significant.

\subsection{Immunogenicity of the Prepared Conjugate}

The immunogenicity of D-LPS-DT conjugate vaccine was analyzed by immunization in mice and compared with D-LPS, DT and normal saline. Table 1 shows anti-LPS antibody titers in immunized mice. Neither DT, nor saline evoked antibodies. As shown in Table 1, two weeks after the first injection, there was no statistically significant difference between antibody levels in mice immunized by D-LPS and D-LPS-DT $(\mathrm{P}<0.01)$. However, after the second and third injections, D-LPS-DT displayed higher titers in IgG and IgM LPS antibodies than D-LPS $(\mathrm{P}<0.01)$. The control groups also indicated the lowest antibody titers. The results of all types of anti-LPS antibodies (total IgG, IgM,
IgA, IgG1, IgG2a, IgG2b and IgG3) after two weeks were also indicated in Figure 3. D-LPS-DT conjugate showed the highest responses among the all types of antibodies. As observed, the anti-LPS IgG antibody was predominantly IgG1 subclass and then IgG3, IgG2a and IgG2b, respectively. Briefly, these results showed that the conjugate vaccine based on LPS from P. aeruginosa and DT raised LPS antibodies.

\section{Discussion}

Pseudomonas aeruginosa is one of the most important causes of hospital acquired infections. Due to the resistance to antibiotic, P. aeruginosa infections are difficult to treat (13). Several approaches have been examined to protect patients against $P$. aeruginosa infections including passive immunization with monoclonal and polyclonal antibody. The most promising vaccines were the LPS based vaccines $(14,15)$. Generally, the conjugate vaccine is preferred over other immunogens because of its safety and its potential to elicit high quantities of protective antibodies. Anti-LPS antibody has been shown to be highly

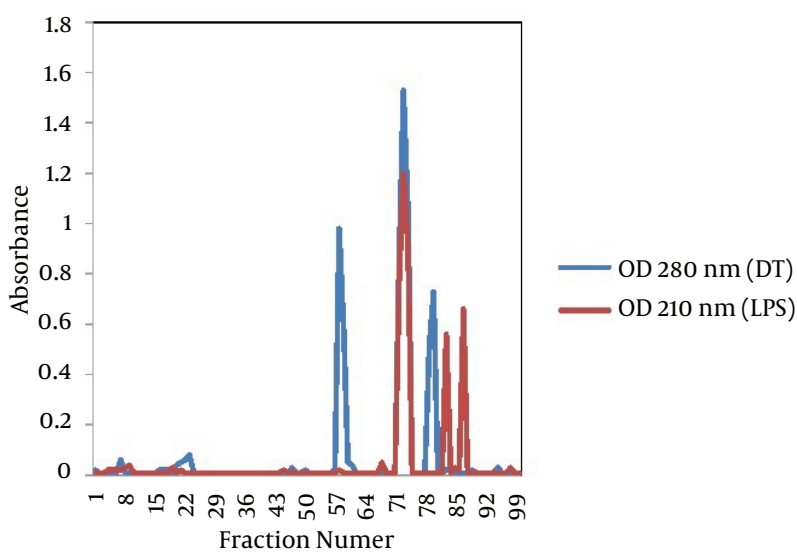

Figure 2. Gel Filtration Profile of D-LPS-DT Conjugate Through a CL-2B Sepharose Column

Table 1. Comparison of LPS Antibodies Elicited by D-LPS-DT, D-LPS, DT and Normal Saline as Control Group in Mice

\begin{tabular}{lccccc}
\hline Immunogen & IgM, d 14 & IgM, d 28 & IgM, d 42 & IgG, d 14 & IgG, d 28 \\
\hline D-LPS-DT & 112 & 250.2 & 679 & $369^{\mathrm{a}}$ & $1140^{\mathrm{a}}$ \\
D-LPS & $100^{\mathrm{a}}$ & $190^{\mathrm{a}}$ & $217^{\mathrm{a}}$ & $100^{\mathrm{a}}$ & $372^{\mathrm{a}}$ \\
DT & 0 & 0 & 0 & 0 & 0 \\
Negative control & 0 & 0 & 0 & 0 & 0 \\
\hline
\end{tabular}

a The mean difference is significant at the 0.01 level. 
A

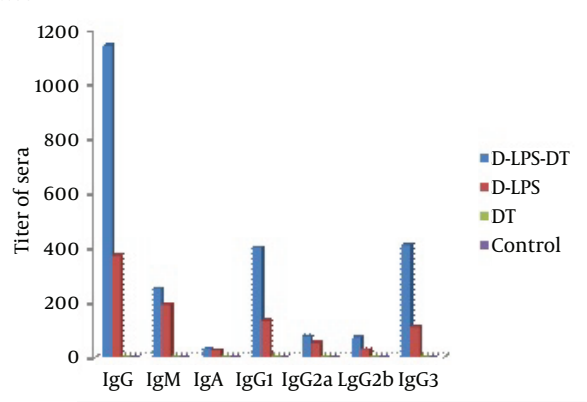

B

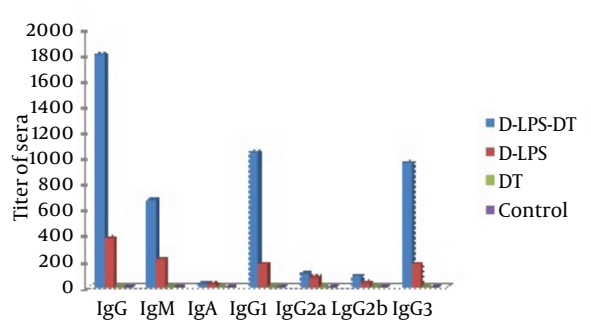

Figure 3. Induction of Anti-LPS Antibodies in BALB/C Mice for Days 28 (A) and 42 (B)

protective against $P$. aeruginosa infections (6). The LPS based conjugate vaccine has stimulated the induction of antibodies against $P$. aeruginosa when tested in burn patients and led to reduction of mortality from P. aeruginosa infection. However, LPS vaccines have not met the approval for routine clinical use because of their toxicity associated with their lipid A fractions (16).

Attempts to apply P. aeruginosa unaltered LPS as a vaccine have been hampered by a high frequency of adverse reactions after immunization and also the need for numerous injections to evoke an optimal immune response (6). Subsequently, subunit vaccines based on the conjugated LPS to P. aeruginosa exotoxin or alginate or isolated flagella have been shown to be less toxic and elicit antibodies in a number of volunteers and patient groups (16). In the current study, LPS from P. aeruginosa PAO-1 was investigated for the preparation of a conjugate vaccine. Conjugation of polysaccharide antigens to carrier proteins has been used to increase their immunogenicity and create effective vaccines (7). This procedure converts polysaccharide from a T-cell-independent antigen into a T-cell-dependent antigen and elicits a higher and booster immune response in animals (17). Several highly immunogenic bacterial proteins have been proposed as carriers for conjugate vaccines, including bacterial pili, outer membrane proteins (OMPs) and excreted toxins of pathogenic bacteria, preferably in toxoid form (8). Meanwhile, some proteins such as tetanus toxoid (TT) and bovine serum albumin (BSA) have been used as carrier proteins to conjugation with O-polysaccharide (O-PS) from P. aeruginosa (18). In this work, we used DT as the carrier protein, because DT is readily available and a part of the pediatric immunizations, which is performed within the frame of Expanded Programme of Immunization of the WHO and UNICEF (17).

The DT has been shown to enhance immunogenicity of polysaccharide vaccines when used as the carrier protein in conjugate vaccine. The D-LPS was conjugated to DT via the amidation method using EDAC as a linker and $\mathrm{ADH}$ as a spacer molecule. The spacing and density of the saccharide on protein are likely to have major impacts on the ability of conjugate to induce an immune response (7). The conjugation molar ratio of LPS to DT was 3:1. The conjugation efficiency was also calculated to be $43 \%$. The titers of all types of antibodies prepared from immunized mice sera with D-LPS-DT vaccine showed significant rise compared to D-LPS. In this research, contrary to other studies which focused on IgG titer, the rate of IgM, IgA and IgG subclasses were also investigated. The results of anti-LPS inductions for total IgG, IgM, IgA, IgG1, IgG2a, IgG2b and IgG3 achieved as D-LPS-DT > D-LPS > DT. The highest antibody titers were obtained with IgG and its subclasses. In fact, in the absence of DT, there was no significant enhancement of immunogenicity with D-LPS. Similar protection has been obtained using LPS-DT conjugate derived from Leptospira interrogans (8).

In summary, conjugation of LPS from P. aeruginosa PAO-1 to DT appears to be promising for production of an LPSbased conjugate vaccine. The D-LPS-DT conjugate vaccine was non-toxic, non-pyrogenic and elicited high levels of IgG and IgM antibodies. These results showed that the constructed D-LPS-DT conjugate vaccine had considerable potential to protect against $P$. aeruginosa infections.

\section{Acknowledgements}

This work was supported by Islamic Azad University, Zanjan Branch, Zanjan, IR Iran.

\section{References}

1. Siegel RE. Emerging gram-negative antibiotic resistance: daunting challenges, declining sensitivities, and dire consequences. Respir Care. 2008;53(4):471-9.

2. Taheri ZM, Shahbazi N, Khoddami M. Genetic Diversity of pseudomonas aeruginosa Strains isolated from Hospitalized patients. Tanaffos. 2008;7(1):32-9.

3. Al-Zeer M, Masoud H. LPS-based conjugate vaccines composed of O-polysaccharide from Pseudomonas aeruginosa IATS 6 and 11 bound to a carrier protein. World J Microbiol Biotechnol . 2007;23(11):1541-9.

4. Donta ST, Peduzzi P, Cross AS, Sadoff J, Haakenson C, Cryz SJ, et al. Immunoprophylaxis against Klebsiella and Pseudomonas aeruginosa Infections. J Infect Dis. 1996;174(3):537-43.

5. Cryz SJJ, Lang AB, Sadoff JC, Germanier R, Furer E. Vaccine potential of Pseudomonas aeruginosa O-polysaccharide-toxin A conjugates. Infect Immun. 1987;55(7):1547-51.

6. Cryz SJ, Sadoff JC, Fiirer E, Germanier R. Pseudomonas aeruginosa Polysaccharide-Tetanus Toxoid Conjugate Vaccine: Safety and Immunogenicity in Humans. J Infect Dis. 1986;154(4):682-8.

7. Robinson A, Hudson MJ, Cranage MP. Vaccine Protocols. 2 ed: Humana press; 2000. 
8. Midwinter A, Faine S, Adler B. Vaccination of mice with lipopolysaccharide (LPS) and LPS-derived immuno-conjugates from Leptospira interrogans. J Med Microbiol. 1990;33(3):199-204.

9. Aspe M, Jensen L, Melegrito J, Sun M. The role of alginate and extracellular DNA in biofilm-meditated Pseudomonas aeruginosa gentamicin resistance. J Exp Microbiol Immunol. 2012;16:42-8.

10. Kashef N. Synthesis and characterization of Pseudomonas aeruginosa alginate-tetanus toxoid conjugate. J Med Microbiol. 2006;55(10):1441-6.

11. Konadu E, Robbins JB, Shiloach J, Bryla DA, Szu SC. Preparation, characterization, and immunological properties in mice of Escherichia coli 0157 O-specific polysaccharide-protein conjugate vaccines. Infect Immun. 1994;62(11):5048-54.

12. Cryz SJ, Furer E, Cross AS, Wegmann A, Germanier R, Sadoff JC. Safety and immunogenicity of a Pseudomonas aeruginosa Opolysaccharide toxin A conjugate vaccine in humans. J Clin Invest. 1987;80(1):51-6.

13. Zeng X, Liu X, Bian J, Pei G, Dai H, Polyak SW, et al. Synergistic effect of 14-alpha-lipoyl andrographolide and various antibiotics on the formation of biofilms and production of exopolysaccharide and pyocyanin by Pseudomonas aeruginosa. Antimicrob Agents Chemother. 2011;55(6):3015-7.

14. Kunert A, Losse J, Gruszin C, Huhn M, Kaendler K, Mikkat S, et al. Immune evasion of the human pathogen Pseudomonas aeruginosa: elongation factor Tuf is a factor $\mathrm{H}$ and plasminogen binding protein. J Immunol. 2007;179(5):2979-88.

15. Cryz SJ, Furer E, Germanier R. Protection against fatal Pseudomonas aeruginosa burn wound sepsis by immunization with lipopolysaccharide and high-molecular-weight polysaccharide. Infect Immun. 1984;43(3):795-9.

16. Baumann U, Mansouri E, von Specht BU. Recombinant OprF-OprI as a vaccine against Pseudomonas aeruginosa infections. Vaccine. 2004;22(7):840-7.

17. Collier L, Balows A, Sussman M. Topley \& Wilson's microbiology and microbial infections. 9 ed: Arnold; 1998.

18. Cryz SJ, Sadoff JC, Fürer E. Octavalent Pseudomonas aeruginosa O-polysaccharide-toxin A conjugate vaccine. Microb Pathog. 1989;6(1):75-80. 